\title{
Sirtuin-mediated nuclear differentiation and programmed degradation in Tetrahymena
}

Kristin M Slade ${ }^{1,2}$, Sydney Freggiaro ${ }^{1}$, Kyle A Cottrell $^{3}$, Joshua J Smith ${ }^{3}$ and Emily A Wiley ${ }^{1 *}$

\begin{abstract}
Background: The $\mathrm{NAD}^{+}$-dependent histone deacetylases, known as "sirtuins", participate in a variety of processes critical for single- and multi-cellular life. Recent studies have elucidated the importance of sirtuin activity in development, aging, and disease; yet, underlying mechanistic pathways are not well understood. Specific sirtuins influence chromatin structure and gene expression, but differences in their pathways as they relate to distinct chromatin functions are just beginning to emerge. To further define the range of global chromatin changes dependent on sirtuins, unique biological features of the ciliated protozoan Tetrahymena thermophila can be exploited. This system offers clear spatial and temporal separation of multiple whole genome restructuring events critical for the life cycle.

Results: Inhibition with nicotinamide revealed that sirtuin deacetylase activity in Tetrahymena cells promotes chromatin condensation during meiotic prophase, differentiation of heterochromatin from euchromatin during development, and chromatin condensation/degradation during programmed nuclear death. We identified a class 1 sirtuin, called Thd14, that resides in mitochondria and nucleoli during vegetative growth, and forms a large subnuclear aggregate in response to prolonged cell starvation that may be peripherally associated with nucleoli. During sexual conjugation and development Thd14 selectively concentrates in the parental nucleus prior to its apoptotic-like degradation.

Conclusions: Sirtuin activity is important for several functionally distinct events requiring global chromatin condensation. Our findings suggest a novel role for sirtuins in promoting programmed pycnosis by acting on chromatin destined for degradation. The sirtuin Thd14, which displays physiological-dependent differential localization within the nucleus, is a candidate for a chromatin condensation enzyme that is coupled to nuclear degradation.
\end{abstract}

Keywords: programmed nuclear degradation, apoptosis, sirtuin, HDAC, Tetrahymena, ciliate, histone deacetylase

\section{Background}

Class III histone deacetylases, known as sirtuins, are a large and ancient family of $\mathrm{NAD}^{+}$-dependent protein deacetylases that regulate a range of cellular processes. These phylogenetically conserved enzymes deacetylate both histone and non-histone targets. Originally based on the founding family member, yeast Sir2, molecular phylogenetic analyses have since revealed five sirtuin subclasses I-IV and U [1], which display diversity in subcellular localization and function $[2,3]$. For example, of

\footnotetext{
* Correspondence: ewiley@jsd.claremont.edu

'Keck Science Department of Claremont McKenna, Pitzer, and Scripps Colleges, W.M. Keck Science Center, 925 N. Mills Ave., Claremont, CA 91711 USA

Full list of author information is available at the end of the article
}

the seven human sirtuin homologs, several reside in the nucleus where they have roles in genomic stability and cell proliferation. Others act in the cytoplasm on cytoskeletal targets or work in mitochondria to regulate energy metabolism and responses to oxidative stress $[4,5]$. Sirtuins in subclass I, which include human SIRT1-3 and yeast Hst2 and Sir2, commonly localize to the nucleus (with exception of Hst2) where they have various chromatin-related functions. For example, Sir2 regulates telomeric, cryptic mating-type, and rDNA silencing [6]. This diverse set of functions underlies numerous links between development, disease, and sirtuin activity reported in recent years $[7,8]$.

Many sirtuin-linked cell abnormalities may relate to their roles in chromatin dynamics. To further probe

\section{() Biomed Central}


these possibilities we turned to the single-celled protozoan Tetrahymena thermophila. This ciliate expresses eleven putative sirtuins, most are more closely related to sirtuins in humans than to those in yeasts [9]. Tetrahymena provides several advantages for chromatin dynamics studies. First, the cells harbor two nuclei with different chromatin characteristics. The "macronucleus" is transcriptionally active and contains primarily euchromatin, but undergoes widespread facultative heterochromatin formation during cell starvation $[10,11]$. Conversely, the "micronucleus" is transcriptionally silent and contains chromatin that is highly condensed into constitutive heterochromatin-like structures throughout vegetative growth [12]. This unique nuclear dimorphism facilitates study of factors that contribute to the differentiation and maintenance of euchromatin and heterochromatin states in the respective nuclei.

Second, nuclear differentiation into the dimorphic micronucleus and macronucleus during sexual conjugation involves multiple processes including DNA replication, DNA fragmentation and elimination, chromatin remodeling/differentiation, and nuclear degradation. These events are easily synchronized in a cell population and occur in a strict temporal order in only a subset of the post-zygotic nuclei (resulting from meiosis, fertilization, and mitosis). Half of the highly condensed, transcriptionally inert postzygotic nuclei differentiate into transcriptionally active, euchromatic nuclei, while the other half remain inert. Another notable feature of Tetrahymena development is the programmed degradation of select nuclei at distinct points in the development pathway. Following meiosis, three of four gametes degrade in the posterior end of the cell. Later, the parental macronucleus degrades as newly differentiating macronuclei become transcriptionally active [12]. The latter degradation mechanism resembles that of caspase-independent apoptosis in higher organisms in several ways, including occurrence of chromatin condensation and production of oligonucleosome sized DNA fragments [13], but lacks other hallmarks such as the phosphorylation of H2A.X [14,15].

In this study we investigated the contribution of sirtuin deacetylase activity to chromatin differentiation and programmed nuclear degradation. We found that nicotinamide, a physiological sirtuin inhibitor $[16,17]$, prevented normal progression of both of these processes. Furthermore, we identified one sirtuin (named "Tetrahymena histone deacetylase 14"; Thd14) that resides in nucleoli, in mitochondria, and in distinct nuclear substructures, all in response to different physiological conditions and stages of the life cycle. Intriguingly, Thd14 accumulated in chromatin-rich regions of the degrading macronucleus during the period of chromatin condensation that precedes global degradation.

\section{Results}

Nicotinamide treatment affects meiotic condensation, nuclear differentiation, and degradation of the macronucleus during development

Phylogenetic analyses previously revealed eleven Tetrahymena proteins that possess sirtuin-type deacetylase domains [9]. This is a larger number than what most other organisms, including mammals, possess. Four of the proteins cluster in sirtuin subclass I, most members of which have nuclear functions. The potential contribution of sirtuin deacetylase activity to nuclear differentiation in Tetrahymena was examined by assessing nuclear development in conjugating cells treated with nicotinamide (NAM), a physiological sirtuin inhibitor $[17,18]$. Major chromatin changes during nuclear differentiation are accompanied by easily-distinguished changes in nuclear morphology. Cells of two different mating types were mixed (time 0 ) and immediately treated with 0,10 , 25 or $50 \mathrm{mM}$ NAM. These concentrations were chosen based on those typically used for yeast and human cells (1-5 mM) and the fact that for Tetrahymena, other histone deacetylase inhibitors must be used at 5-10-fold higher concentrations for effects comparable to mammalian cells [19]. Nuclear morphology in NAM-treated conjugating pairs was compared to that of conjugating control cells (treated with buffer only), by DAPI staining and fluorescence microscopy at 2-3 hour intervals. Cells treated with $50 \mathrm{mM}$ NAM arrested four hours into conjugation at meiotic prophase I (Figures $1 \mathrm{a}$ and $1 \mathrm{~b})$. At this stage micronuclei have drastically elongated and chromosomes have decondensed and assumed a "bouquet-like" arrangement [20] (Figure 1c, right panel). Based on the size and shape of prophase micronuclei in the NAM-arrested cells, the majority appeared to be in zygotene (stage III) and pachytene (stage IV) [21], even 24 hours later (Figure 1b). Cells treated with $10 \mathrm{mM}$ (data not shown) and $25 \mathrm{mM}$ NAM were able to progress through meiosis (Figure 1b) but displayed other downstream phenotypes described below. These phenotypes were detected in a greater fraction of cells treated with $25 \mathrm{mM}$ NAM; thus, this concentration was used for further analyses.

Normally after fertilization, Tetrahymena zygotic nuclei undergo two mitoses. Two of the four resulting nuclei remain highly condensed, transcriptionally inert micronuclei, while the other two differentiate into new transcriptionally active macronuclei, called "anlagen" (Figure 1a). NAM-treated cells that were able to progress past meiosis contained 1-7 additional nuclei per conjugating pair after 24 hours, compared to untreated cells that had reached "endpoint" nuclear configuration by hour 16 (compare Figures 1c "Endpoint" and 1d). The additional nuclei in nicotinamide-treated cells likely 


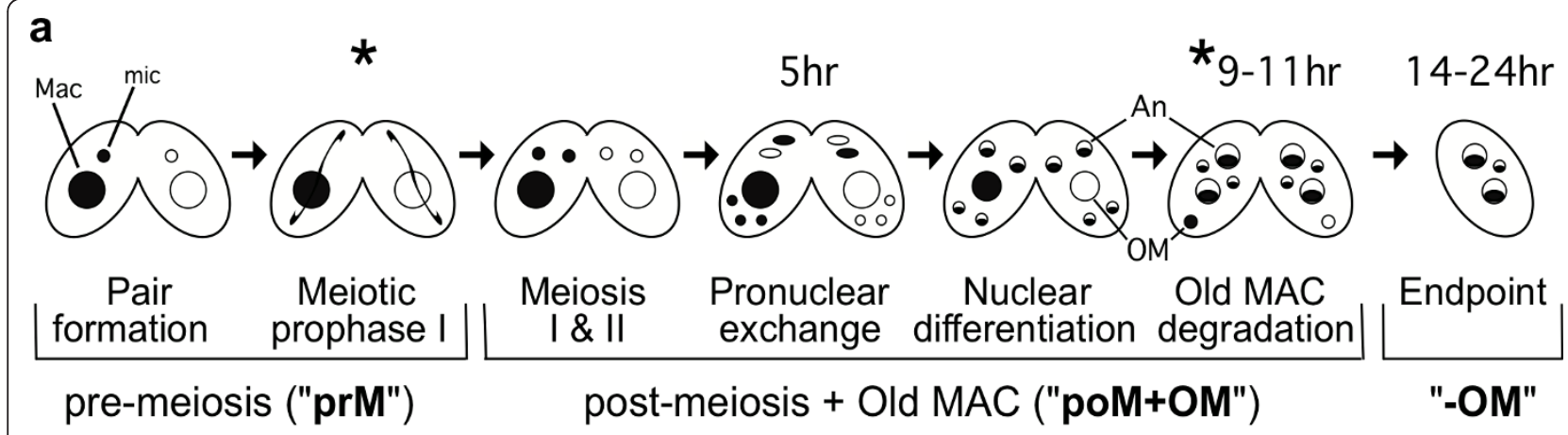

b

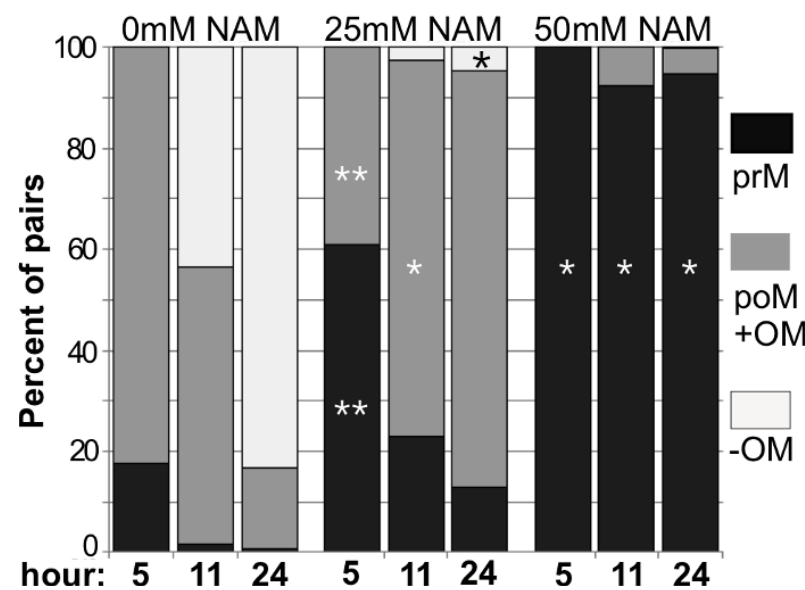

C

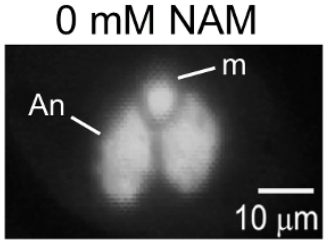

Endpoint

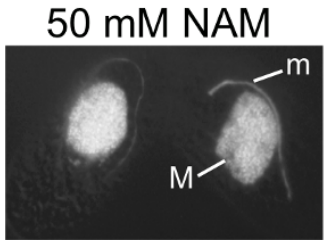

prM arrest

d
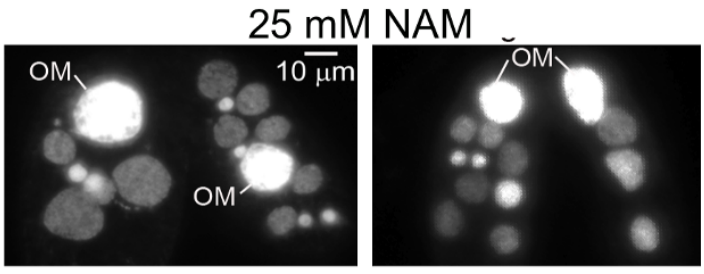

Figure 1 Nicotinamide treatment causes arrest in meiotic prophase and prevents macronuclear degradation. a Schematic of nuclear events in conjugation. Macronuclei are represented as large spheres, micronuclei as small spheres. Two mating types (white and shaded) were mixed to initiate conjugation. Asterisks represent the stages of arrest observed after nicotinamide treatment (coinciding with images in parts $c$ and d). Hours represent times at which untreated conjugating cells ( $0 \mathrm{mM} N A M)$ reach the given stage. Labels below the brackets indicate stages assessed in part b that correspond to different shades in the bar graph. "pOM+OM" are post-meiotic stages that still have a parental macronucleus (old macronucleus; $\mathrm{OM}$ ); "-OM" is any stage after loss of the old macronucleus. An, anlagen (new macronuclei). b The average percentage of conjugating pairs at each of the three stages illustrated in part a by brackets: "PrM", cells in pre-meiosis (black); "pOM+OM", cells that contain an old macronucleus (dark gray); "-OM", cells that have lost the old macronucleus (light gray). Nicotinamide was added just after mixing (time zero of conjugation) and analyzed at 5, 11, and 24 hrs postmixing. At least 200 cells over two separate trials were counted for each time point and NAM concentration (each bar). ${ }^{* *} \mathrm{P}<0.01$ in a t-test when compared to $0 \mathrm{mM}$ NAM at the same time point and stage of meiosis; *P < 0.05. c Representative images of DAPl-stained wild-type (0 mM NAM) and $50 \mathrm{mM}$ NAM treated cells analyzed at 24-hrs into conjugation. M, macronucleus; An, anlagen; m, micronucleus. d DAPI images of 25 mM NAM-treated cells analyzed 24 hrs into conjugation. "OM", old (parental) macronucleus.

resulted from one of two events: failure of three gamete pronuclei to degrade after meiosis, or additional mitoses of zygotic nuclei after fertilization. The former possibility was supported based on the following: 1) gamete pronuclei were rarely observed at the extreme posterior end of the cells where degradation occurs; 2) never were there fewer than four equally-sized nuclei observed in any cell of a pair during the early conjugation stages (normally three nuclei would appear much smaller when degrading at the posterior end; Figure 1a).

Later in conjugation, the extra nuclei appeared to differentiate judging from nucleus size and intensity of
DAPI staining (micronuclear DNA is entirely packaged into unacetylated heterochromatin and stains more intensely with DAPI, while anlagen chromatin decondenses, becomes highly acetylated, and stains less intensely with DAPI). The majority of nuclei in each cell were enlarged with diffuse chromatin, typical of anlagen morphology (Figure 1d). To further examine the differentiated status of chromatin, nuclei in NAM-treated conjugating cells were probed for the presence of the micronucleus-specific linker histone (MLH) by immunofluorescence with $\alpha-M L H$ antiserum. MLH, a hallmark of silent micronuclear heterochromatin, is selectively 
eliminated in nuclei destined to become new macronuclei when euchromatin differentiation and transcriptional activity is initiated [22]. Like all normal postzygotic nuclei, NAM-treated nuclei contained MLH prior to differentiation (Figure 2a, $10 \mathrm{hrs}$ ). However, by 24 hrs after mixing, most cells ( 90\%) contained no MLH-positive nuclei; in approximately $10 \%$ of the cells one MLH-positive nucleus was observed (shown for signal comparison, Figure 2a, 24 hrs.). In contrast, untreated cells each contained one clear MLH-positive micronucleus (and two MLH-negative anlagen), reflecting normal development at $24 \mathrm{hrs}$ after mixing. The loss of MLH in most nuclei of NAM-treated cells indicated that the extra nuclei were differentiating primarily into new macronuclei containing euchromatin (anlagen) at a ratio of 3-6 anlagen:1 micronucleus/cell (opposed to the normal 2 anlagen:1 micronucleus/cell). This interpretation was supported by the swollen appearance and weaker DAPI staining of these nuclei, two hallmarks of new macronuclei. Moreover, immunofluorescence studies showed that during their development, 90\% of the nuclei in NAM-treated cells acquired at least two other macronucleus-specific marks that appear in developing anlagen: the nucleolar protein Nop52 (Figure 2b), and

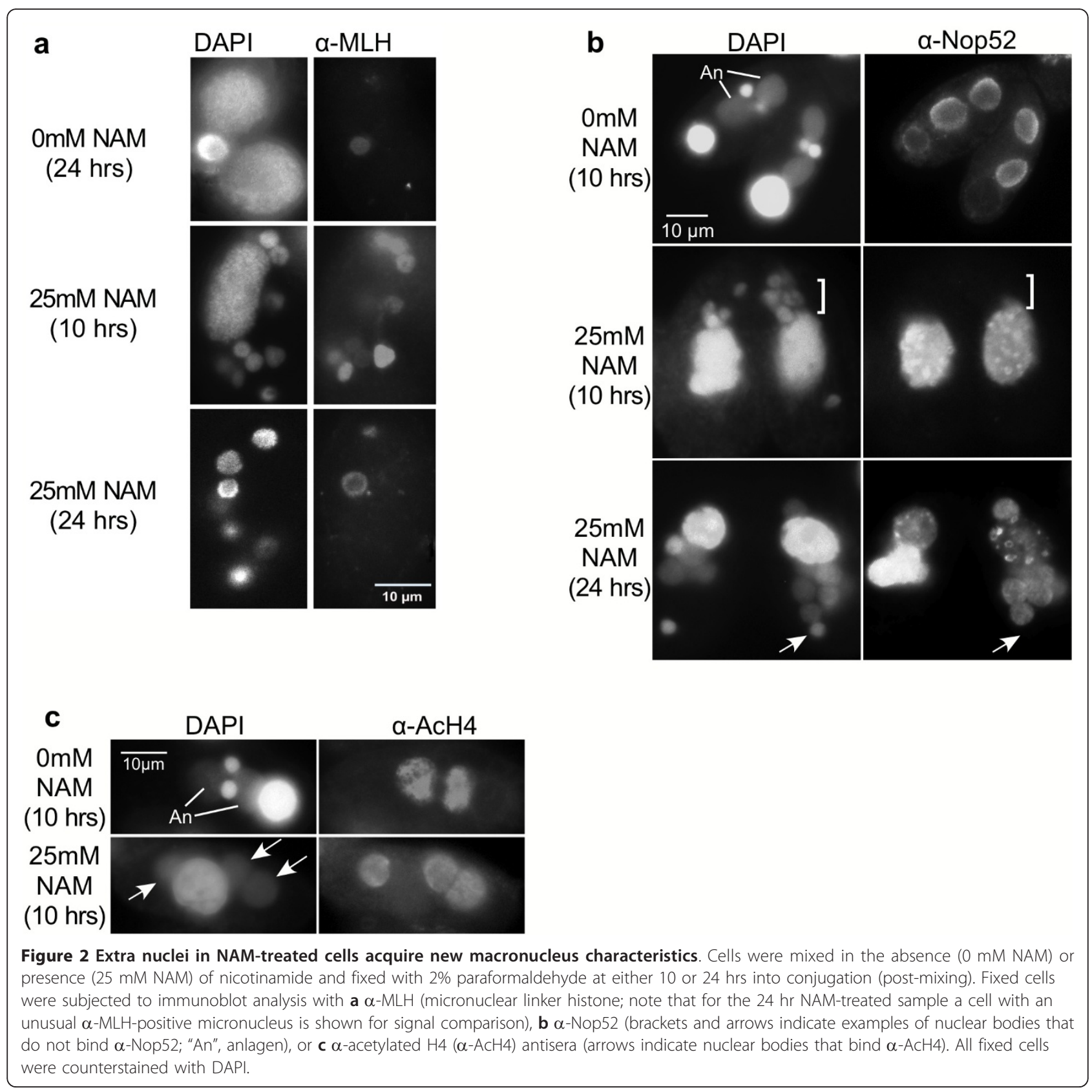


acetylated histones (Figure 2c). Together, these results suggest that sirtuin activity is necessary for the maintenance of MLH and for the development of heterochromatin in half of the post-zygotic nuclei (those destined to differentiate into transcriptionally silent micronuclei). Furthermore, chromatin in additional nuclei (from sirtuin inhibition) appeared to differentiate toward transcriptional competency.

During the nuclear differentiation process, the old parental macronucleus migrates to the posterior end of the cell (Figure 1a) and degrades by a regulated mechanism that is coordinated with other developmental events. The mechanism shares features with apoptosis (chromatin condensation and oligonucleosome laddering) and autophagy (fusion of mitochondria and formation of an autophagosome) [13]. In our experiments, the old parental macronucleus failed to degrade in the majority of NAM-treated pairs. Cells retained their parental macronucleus even 24 hrs after mixing when new macronuclei had at least partially differentiated (Figure 1d), suggesting that sirtuin deacetylase activity was necessary for normal macronuclear degradation, which initiates prior to this stage. In addition, a larger fraction of cells retained their parental macronucleus if treated with NAM earlier in conjugation than shortly before its programmed degradation (see Additional File 1). Treatment immediately prior to the start of macronuclear degradation ( $\sim 8 \mathrm{hrs}$ ) resulted in only $\sim 33 \%$ cells retaining the old macronucleus ( $\sim 2 \mathrm{X}$ more than untreated cells), compared to $\sim 79 \%$ when treated at 2 hours post-mixing $(\sim 5 \mathrm{X}$ more than untreated cells).

To test whether NAM treatment compromised DNA degradation, conjugating pairs were subjected to a TUNEL (terminal deoxynucleotidyl transferase dUTP nick end labeling) assay to detect fragmented DNA. As expected, parental macronuclei in untreated conjugating cells were TUNEL positive $10 \mathrm{hrs}$ into conjugation (Figure 3). In contrast, NAM-treated cells at the same stage with (obvious anlagen) contained a TUNEL-negative parental macronucleus. This result supported the idea that parental macronuclear DNA failed to degrade in sirtuin-inhibited cells. Occasionally, a single TUNEL positive micronucleus was observed later in conjugation, likely one that normally degrades at the end of conjugation (refer to Figure 1a). Here, this served as a positive signal comparison for "TUNEL-negative" nuclei (Figure 3, see arrow).

These results, which suggest a role for sirtuins in apoptotic-like nuclear degradation, prompted us to identify specific sirtuins that may contribute to the degradation process. The putative sirtuin named Tetrahymena Histone Deacetylase 14 (Thd14) was identified for further investigation due to its localization to the parental macronucleus in preliminary studies.

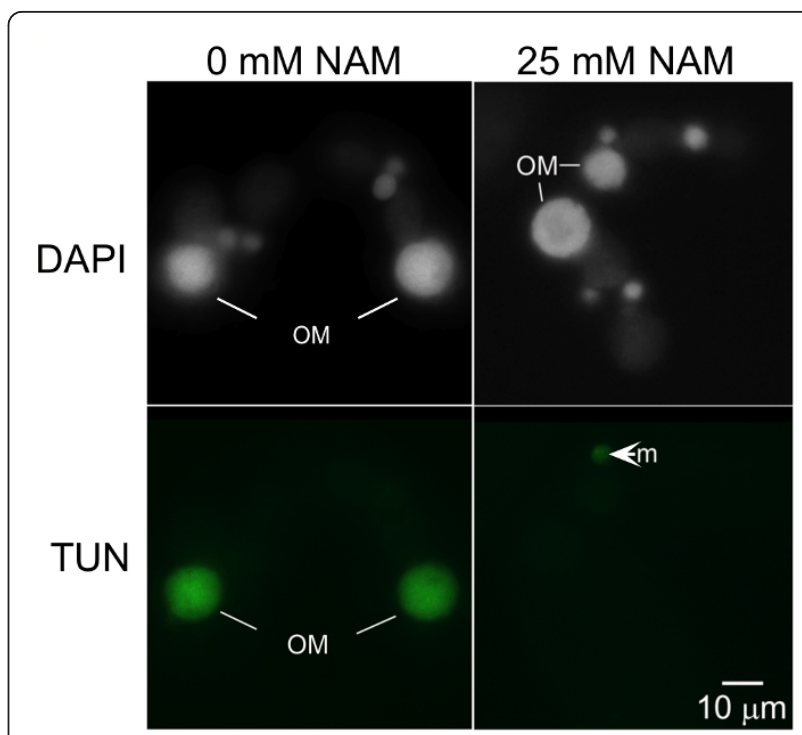

Figure 3 Nicotinamide prevents degradation of the parental macronucleus during conjugation. Just prior to mixing, cells were exposed to either $0 \mathrm{mM}$ or $25 \mathrm{mM}$ nicotinamide (NAM). At $10 \mathrm{hrs}$ into conjugation (post-mixing), the cells were fixed in $2 \%$ paraformaldehyde, exposed to terminal deoxynucleotidyl transferase dUTP nick end labeling (TUNEL) reagent to assay for degraded DNA, and stained with DAPI prior to imaging. In cells exposed to NAM, the old macronuclei "OM" were TUNEL negative, while the OM of nontreated cells were TUNEL positive (green). "m", micronucleus. Note: A cell with an unusual TUNEL-positive micronucleus is shown for signal comparison in the NAM-treated sample.

\section{Thd 14 resembles a class $1 \mathrm{~b}$ sirtuin}

Amino acid sequence comparisons of open reading frames predicted by the Tetrahymena Genome Database (TGD; http://www.ciliate.org) identified gene (TTHERM_00526990), hereafter called THD14, as one of eleven putative sirtuins encoded in the genome [9]. A phylogenetic tree constructed with all human, yeast, and putative Tetrahymena sirtuins showed that Thd14 has strongest similarity to the class 1 sirtuins, along with three other Tetrahymena proteins (Figure 4a). Amino acid alignments between the sirtuin core domain of Thd14 and those of human class I sirtuins (SIRT1, SIRT2, and SIRT3) show strong homology (SIRT1: 45\% identical, 62\% similar; SIRT2: 44\% identical, 61\% similar; SIRT3: 40\% identical, 60\% similar; see Additional File 2 for a multiple sequence alignment).

Multiple Expressed Sequence Tags (ESTs) spanned the majority of the computationally-predicted coding sequence for Thd14 (Tetrahymena Genome Database; http://www.ciliate.org). The expected coding sequence was confirmed using reverse transcriptase-PCR to amplify cDNA with primers spanning the predicted START and STOP codons, followed by cloning and sequencing of the products. Resulting THD14 cDNA sequence was consistent with the computationally 

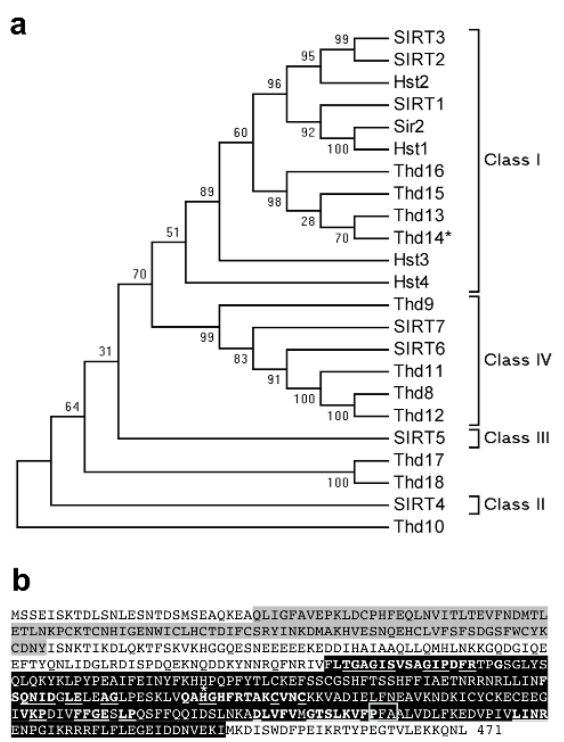

c
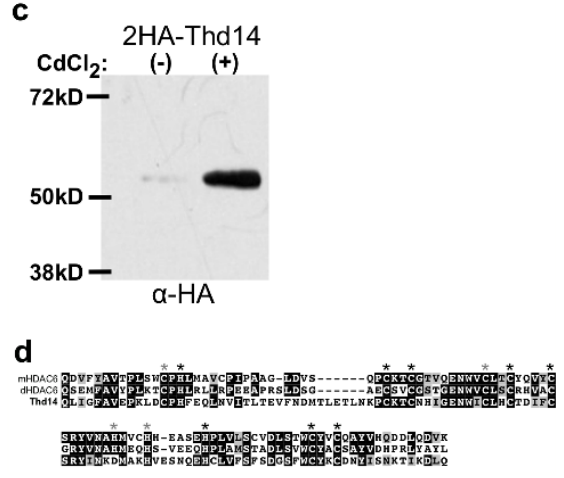

Figure 4 Thd 14 resembles a class $1 \mathrm{~b}$ sirtuin. a Phylogenetic tree comparing the primary structure of the $T$. thermophila sirtuins including Thd14 (asterisk) with that of the yeast sirtuins (Sir2 and Hst1-4) and the seven human sirtuins (SIRT1-7) (unweighted-pair group methods using average linkages). The scale (x-axis) represents evolutionary distance as calculated using the Poisson correction method, with the units of number of amino acid substitutions per site. $\mathbf{b}$ Amino acid sequence of Thd14 translated from the gene sequence. The predicted zinc finger domain is highlighted in grey and the sirtuin domain is highlighted in black. The motifs of conserved amino acids present within the sirtuin core domain are in boldface type. Underlined letters indicate intraclass-conserved residues, used for sirtuin identification. The asterisk denotes the critical catalytic histidine residue in the ' $\mathrm{HG}$ ' motif that is strictly conserved in all known sirtuins. Boxed is a sub-motif specific to class $1 \mathrm{~b}$ sirtuins. c Cells expressing HA-Thd 14 were grown in the absence "(-)"or presence "(+)" of $1 \mathrm{\mu g} / \mathrm{mL} \mathrm{CdCl}$ for 2 hrs to induce expression from the MTT1 promoter. Total cellular proteins were resolved by SDS-PAGE, transferred to nitrocellulose membrane, and subjected to immunoblot analysis using $\alpha-\mathrm{HA}$ antiserum. $\mathbf{d}$ Amino acid alignment of the UBP-type zinc-finger domain from Thd14 with similar domains from murine and Drosophila HDAC6 (mHDAC6 and dHDAC, respectively). Asterisks mark conserved Cys and His residues; black asterisks denote those in HDAC6 that are essential for ubiquitin binding [24]. Conserved residues are shaded in black (for identical residues) and grey (for chemically similar residues). predicted coding sequence. The gene contains one intron and encodes a 471 amino acid protein with two domains: a sirtuin deacetylase domain and a UBP-like zinc finger domain (Figure $4 \mathrm{~b}$ ). The predicted protein molecular mass of $54.1 \mathrm{kD}$ was experimentally confirmed by immunoblot analysis of lysates from cells expressing $2 \times$ HA epitope-tagged Thd14. Anti-HA antiserum detected a protein of $\sim 57 \mathrm{kD}(54 \mathrm{kD}$ plus $\sim 3 \mathrm{kD}$ $2 \times$ HA tag) according to its mobility in SDS-PAGE (Figure 4c).

Computational analysis of Thd14 amino acid sequence revealed a sirtuin "core domain" (Figure 4b, black shading) with typical sirtuin motifs (Figure 4b, bold white lettering) and highly conserved (underlined) catalytic domain residues [1,9]. Importantly, Thd 14 contains the critical catalytic histidine residue in the 'HG' motif that is strictly conserved in all known sirtuins (asterisk, Figure 4b). Immunoprecipitated HAThd14 catalyzed the deacetylation of acetylated Nterminal histone peptide substrates, thus confirming it as a functional histone deacetylase (Additional File 3). The presence of specific intraclass-conserved residues further supported assignment of Thd14 as a class I sirtuin. In particular, the PFA sub-motif is specific to class Ib enzymes, as is the alanine preceding the HG motif (Figure 4b, boxed) [1].

One unique feature of Thd14 is an amino terminal zinc-finger domain of the ubiquitin protease (UBP)-type superfamily, commonly found on ubiquitin hydrolase enzymes (Figure $4 \mathrm{~b}$, grey shading). Interestingly, the highest domain homology is with a UBP-type domain found on the murine class II histone deacetylase HDAC6 (36\% identical, 55\% similar; Figure 4d). Named a Polyubiquitin Associated Zinc finger (PAZ) domain, it coordinates $3 \overline{\mathrm{Zn}}^{2+}$ ions to bind with high affinity to polyubiquitin protein modifications [23-25]. This domain on Thd14 contains 11 out of the 12 highly conserved cysteine and histidine residues used to coordinate the $\mathrm{Zn}^{2+}$ ions (Figure $4 \mathrm{~d}$, all asterisks). Of those residues, the domain on Thd14 contains all that are absolutely required for ubiquitin binding (2 histidines and 6 cysteines) [24] (Figure 4d, indicated by black asterisks). Thd14 is the only one of the eleven Tetrahymena sirtuins that contains a PAZ domain.

\section{THD14 transcription peaks in early conjugation}

THD14 expression throughout the Tetrahymena life cycle was examined. Two different mating types were starved (CU428 and SB1969), mixed to initiate conjugation, and allowed to conjugate over a 24-hour period. Population synchrony was monitored at regular intervals by determining the fraction of cells in each developmental stage (Figure 5a). Throughout the entire conjugation time course, 60 to $70 \%$ of the cells were tightly 

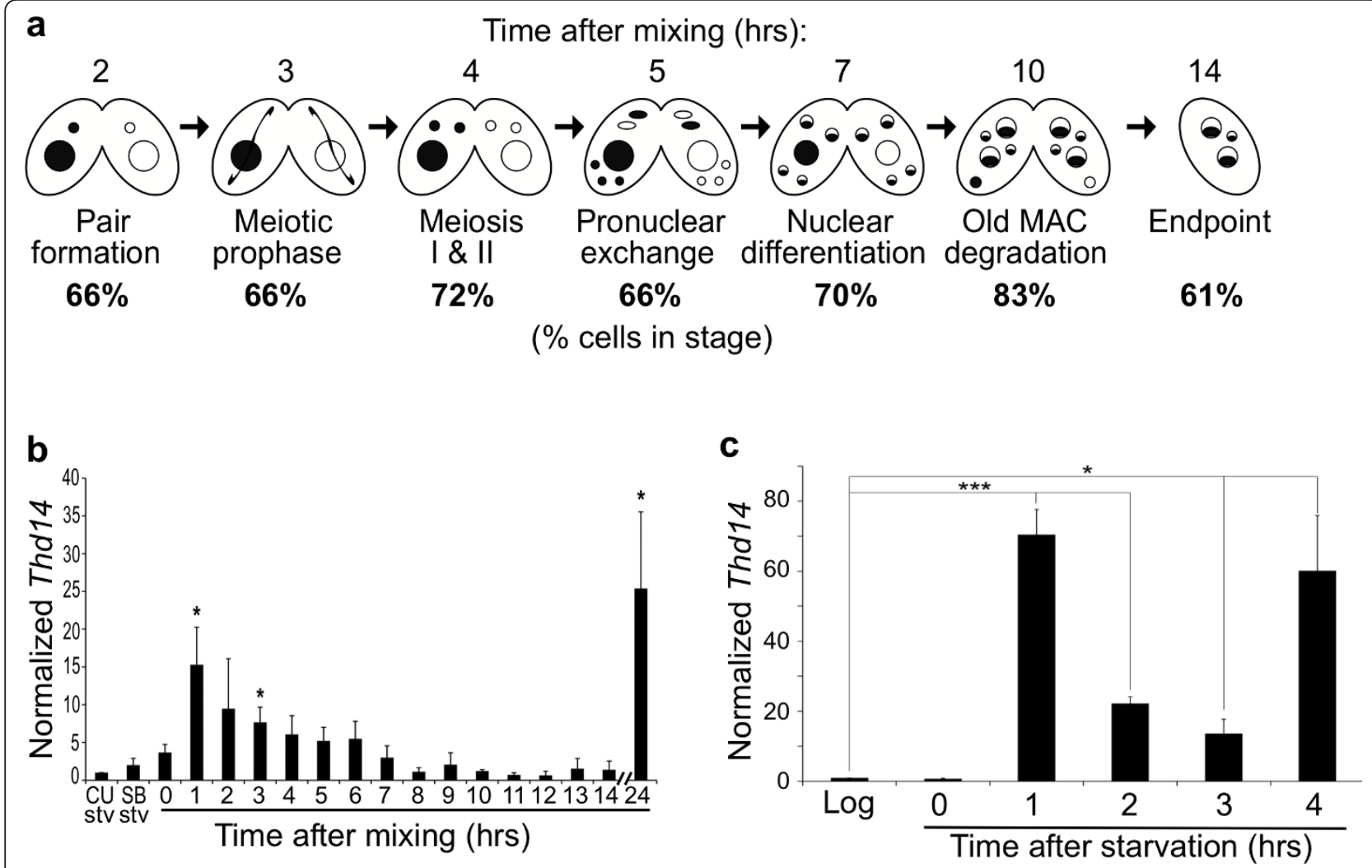

Figure 5 Thd14 expression peaks in pre-meiotic stages. a Synchrony of conjugating cells used for cDNA analyses. Cells of two different mating types were mixed to initiate conjugation, and samples of the conjugating culture were dried and stained with DAPI for visualization of stages by fluorescence microscopy every hour. Hours post mixing are indicated ("hrs") above the corresponding stages illustrated. The percentage of conjugating pairs in each stage at the corresponding time is indicated below the diagram. The last stage is percentage of single cells. b Relative THD14 expression of starved CU428 (CU Stv) and SB1969 (SB Stv) as well as during conjugation (0-14 and 24 hours after mixing) was determined using quantitative real-time PCR. THD14 expression was normalized to CYP1 expression (THD14/CYP1) and the levels of THD14 expression were set relative to the starved CU428 (normalized values of each sample/CU428). Error bars represent the standard deviation of three separate trials and asterisks indicate time points that had a significant $(p<0.05)$ increase in expression of THD14 compared to either the starved CU428 cells (CU Stv) or immediately after mixing (0 hrs). THD14 mRNA levels increased early in conjugation at (1-3 hours) and late (24 hours) after completion of conjugation. c Relative THD14 expression of logarithmically growing CU428 ("Log") as well as during starvation (hours 0 through 4) was determined using quantitative real-time PCR. THD14 expression was normalized to that of BTU1 and the levels of THD14 expression were set relative to logarithmically growing cells. Error bars represent the standard deviation of three separate trials and asterisks indicate time points that had a significant ${ }^{*} p<0.05,{ }^{* *} p<0.005$ ) increase in expression of THD14 compared to logarithmically growing cells.

synchronized and an additional 20 to $25 \%$ of the culture was within 60 minutes of this primary synchronized population. Reverse transcriptase-PCR (data not shown) and $\mathrm{qRT}$-PCR using cDNA made from the conjugating cells at 1-hour intervals (and from mid-logarithmic growing cells) was performed. THD14 transcription was moderate in growing and 24-hour starved cells and elevated in early (pre-meiotic) stages of conjugation. Then, Thd14 expression slowly decreased over subsequent intervals, except at $24 \mathrm{hrs}$ after mixing (once cells have all achieved "endpoint"), where the highest expression level was observed (Figure 5b). These results were consistent with those from microarray experiments, which also show that the 24 hour peak initiates at $18 \mathrm{hrs}$ http://tged.ihb.ac.cn[26]. Together, these results suggest that THD14 transcription is regulated according to physiological state and developmental stage, and raises the possibility of a role for this sirtuin in pre-meiotic events. In other cell types, select sirtuin activities are induced by starvation. To examine Thd14 expression during starvation, mRNA was quantified by qRT-PCR over a 4 hour period following nutrient depletion. Relative to vegetatively growing cells, a dramatic increase in expression was observed immediately with starvation initiation, and levels remained high over the interval tested (Figure 5c). This result raised the possibility that Thd14 has a starvation-specific function that is rapidly induced with nutrient depletion. 
Thd14 is in mitochondria and nucleoli during vegetative growth, and aggregates during starvation

To examine localization of Thd14, Green Fluorescent Protein (GFP) was fused to the amino terminus. The fusion gene, GFP-THD14, was carried on a high-copy Tetrahymena rDNA vector [27] and expressed from the inducible metallothionein 1 (MTT1) promoter (Figure 6a) $[27,28]$. In mid-logarithmic growing cells, GFPThd14 was detected in mitochondria, which were marked with fluorescent Mitrotracker dye (Figure 6b). GFP-Thd14 was also detected in the multiple nucleoli positioned around the macronuclear periphery $(\sim 90$ nucleoli per nucleus; Figure 6c), which were illuminated by differential interference microscopy (labeled "nu" on DIC image). Food vacuoles in the cytoplasm, a common fluorescent artifact from over-expression of GFP tagged proteins (shown by "GFP con/DAPI" panel), were ignored in this analysis. Consistent with nucleolar localization, immunofluorescence revealed that the nucleolar protein Nop52 [29] co-localized to regions containing GFP-Thd14 (Figure 6d). Furthermore, these regions with GFP-Thd14 stained only weakly with DAPI, a common characteristic of nucleoli.

We next tested whether GFP-Thd14 remained in nucleoli under conditions that alter rDNA metabolism. With prolonged nutrient starvation, Tetrahymena nucleoli aggregate to form larger masses around the nuclear periphery [30] coincident with a global decrease in transcription of rDNA and many protein-coding genes. After 18-20 hours of starvation, GFP-Thd14 was still present in the nucleoli in the majority of cells. However, the most intense fluorescence concentrated in a single large aggregate within the macronucleus (Figure 7a) that was visible by 30 minutes after nutrient depletion (quickly following the increase in THD14 expression; Figure 5c), and reached maximum size 4 hours later. Of the cells showing nucleolar fluorescence around the periphery $(\sim 85 \%)$, approximately half $(45 / 96)$ contained the larger aggregate. The aggregate of fluorescence appeared as a clustering of multiple small rings, suggesting sub-structural organization of Thd14 within the larger aggregate (Figure 7b). As well, DAPI staining was weaker or absent from the concentration of GFPThd14. DIC imaging mapped Thd14 aggregates to distinct structures within the nucleus (Figure $7 \mathrm{~b}$, right panel). Immunofluorescence with anti-Nop52 antiserum revealed that Nop52 ringed the entire Thd14 aggregate (Figure 7c) indicating a relationship to nucleoli.

Thd14 concentrates inside the macronucleus immediately prior to programmed degradation

To assess Thd14 distribution during development and nuclear differentiation, cells expressing GFP-Thd14 were

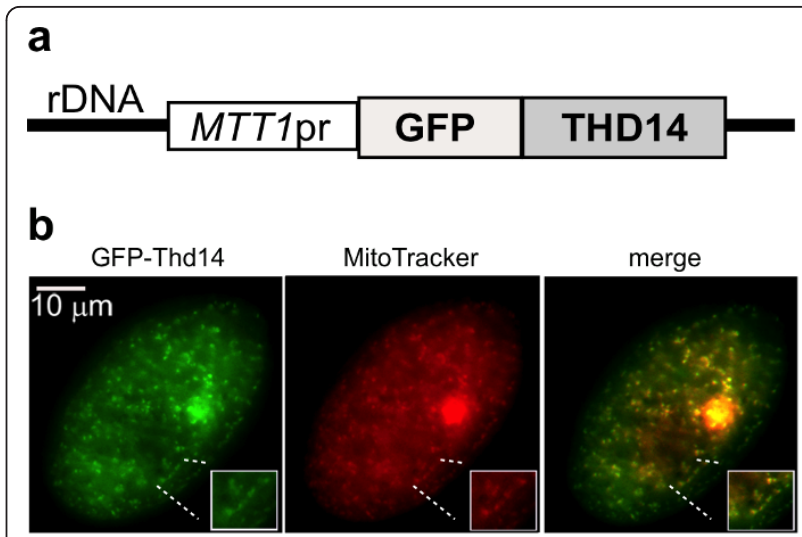

C $\mathrm{DIC}$
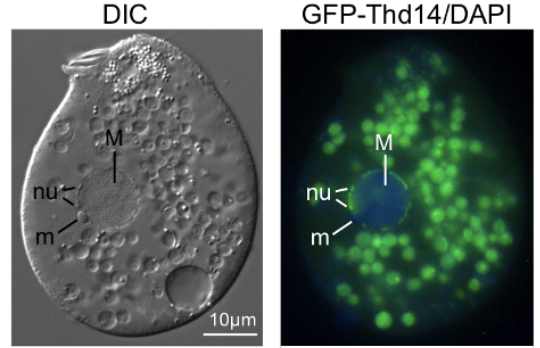

d
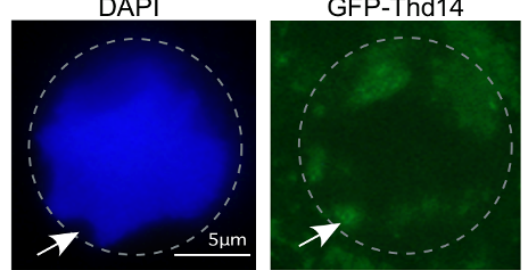

GFP con/DAPI

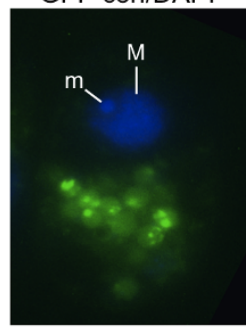

a-Nop52

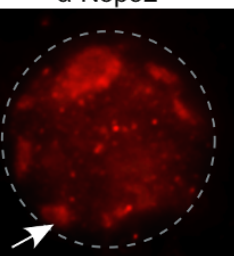

Figure 6 Thd 14 resides in mitochondria and nucleoli during growth. a Line drawing schematic of the construct engineered on an rDNA vector for expression of GFP-Thd14 fusion protein from the metallothioneine promoter (MTT1pr). During conjugation, the vector is processed and amplified as a small linear rDNA chromosome. $\mathbf{b}$ Fluorescence microscopy image of a live cell showing GFP-Thd14 localizing to mitochondria (stained with Mitotracker) near the surface of the cell. Inlays show magnification of one mitochondrialdense region. The larger region of intense staining is rarely observed in other cells and thus considered an artifact. c Differential interference contrast (DIC) imaging of a live cell in vegetative growth shows locations of nuclear structures, including the micronucleus (m) and multiple nucleoli (nu) positioned around the macronuclear (M) periphery. Fluorescence microscopy shows a merge of DAPI imaging with GFP-Thd14 in nucleoli (center panel). "GFP con/DAPI" is the GFP only control (no fusion) with a DAPI image overlay. Green spheres outside the nucleus are probably food vacuoles, a common artifact observed with high GFP-protein expression. Live cells were concentrated and mounted in $2 \%$ methylcellulose for observation. $\mathbf{d}$ Fluorescence microscopy on a paraformaldehyde-fixed cell nucleus (bounded by dashed circle) showing co-localization of GFP-Thd14 and Nop52 by immunofluorescence in regions staining poorly with DAPI (arrow indicates an example). 

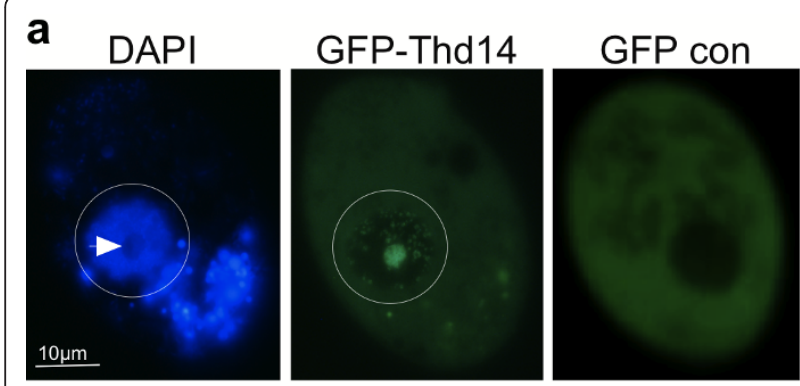

b
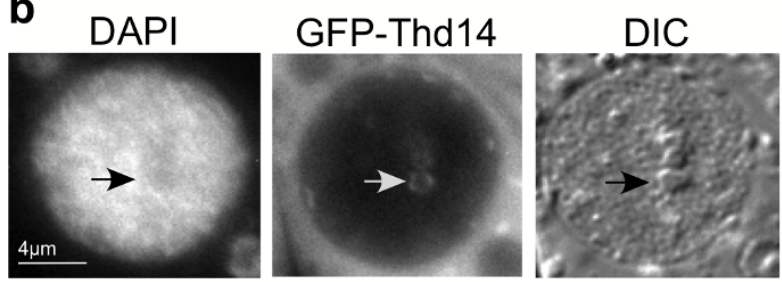

c
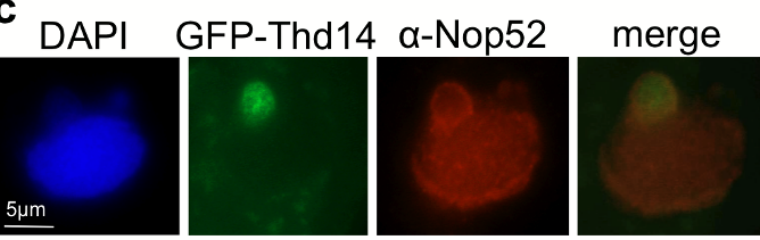

Figure 7 Thd14 resides in a large aggregate during starvation. a A starved live cell expressing GFP-Thd14 visualized by fluorescence microscopy. The macronucleus is within the circled regions. Staining outside the nucleus is in food vacuoles. White arrow points to a DAPI poor region where GFP-Thd14 aggregates. "GFP con" is the negative control expressing only GFP (no fusion with Thd14). b DAPI, GFP and DIC images of a macronucleus in a live cell. Masses inside the macronucleus observed by DIC microscopy (arrow) are ringed by GFP-Thd14 and stain weakly with DAPI, both observed by fluorescence imaging. c A macronucleus showing Nop52 ringing around the large aggregate of GFP-Thd14 by immunofluorescence.

starved and mixed with wild type cells of a different mating type to initiate sexual conjugation. Samples of conjugating pairs were examined by fluorescence microscopy at regular intervals throughout the course of conjugation (16 hours) and 24 hours after initiation (Figure $8 \mathrm{a})$. In early conjugation (2-5 hrs, micronuclear meiosis and mitosis) the rings in the GFP-Thd14 focus became more pronounced, then diffused throughout the macronucleus. By mid-conjugation (5-8 hrs) at stages immediately preceding old macronucleus pycnosis and degradation, GFP-Thd14 became increasingly and selectively concentrated within the old macronucleus, but remained absent from the young, developing new macronuclei (Figure 8a). Within the degrading nucleus, Thd14 localized to the area occupied by pycnotic heterochromatin within the boundary of the nuclear envelope as revealed by DIC microscopy (Figure $8 \mathrm{~b}$ ).

\section{Discussion}

Sirtuins are known to influence chromatin dynamics related to gene silencing, DNA repair, and maintenance of chromosome structural features such as telomeres. In this study we examined processes involving chromatin dynamics that are easily synchronized and monitored using Tetrahymena conjugation. Sirtuin inhibition with nicotinamide (NAM) affected three events requiring significant changes to global chromatin structure: (1) meiotic chromosome condensation, (2) differentiation of transcriptionally inert nuclei, and (3) programmed nuclear degradation.

During meiotic prophase, the germline micronucleus elongates $\sim 50$-fold resulting in a string-like "crescent" nucleus. Chromosomes decondense and assume a bouquet-like formation for homologous pairing and recombination, then re-condense prior to metaphase I [31,32]. With $50 \mathrm{mM}$ NAM treatment, decondensation and elongation was unaffected (normal numbers of fully elongated crescents were observed), but re-condensation appeared incomplete; the majority of cells arrested with partially elongated micronuclei (Figure 1b). The class I sirtuin, SIRT2, was previously implicated in promoting chromosome condensation during mitosis in human cells by deacetylating histone H4 [33]. However, evidence for sirtuin roles in meiotic chromatin dynamics was lacking prior to this study.

Cells in a lower NAM concentration proceeded through meiosis, but displayed other chromatin differentiation defects. First, more than four post-zygotic nuclei were observed in most cells. Of these nuclei, all (or most in some cells) initiated new macronucleus chromatin development involving loss of micronuclear linker histone (MLH) (Figure 2a), and gain of both Nop52 (Figure 2b) and histone acetylation (Figure 2c). The ratio of differentiating macronuclei to micronuclei was 3-6:1 instead of the normal 2:1 in most pairs (Figures $1 \mathrm{~d}$ and 2-all parts). These data suggest that sirtuins normally promote the retention of MLH and development of heterochromatin in micronucleus-destined nuclei. Our observations suggest that the abnormally large number of nuclei in sirtuininhibited cells resulted from failure of gamete degradation prior to fertilization ( 3 of the 4 gametes normally degrade), an aberrant event that could be mechanistically related to the failure of parental macronuclear degradation observed later in conjugation. This idea is consistent with a previous observation that halting macronuclear degradation with PI-3 kinase inhibitors led to the retention and reprogramming of pronuclei to differentiate into micro- and macronuclei [34]. Our results indicate that the retained extra nuclei are capable of differentiating from transcriptionally silent to transcriptionally active nuclei, and that this pathway is chosen (over maintenance of the silent state) in the absence of sirtuin activity. 


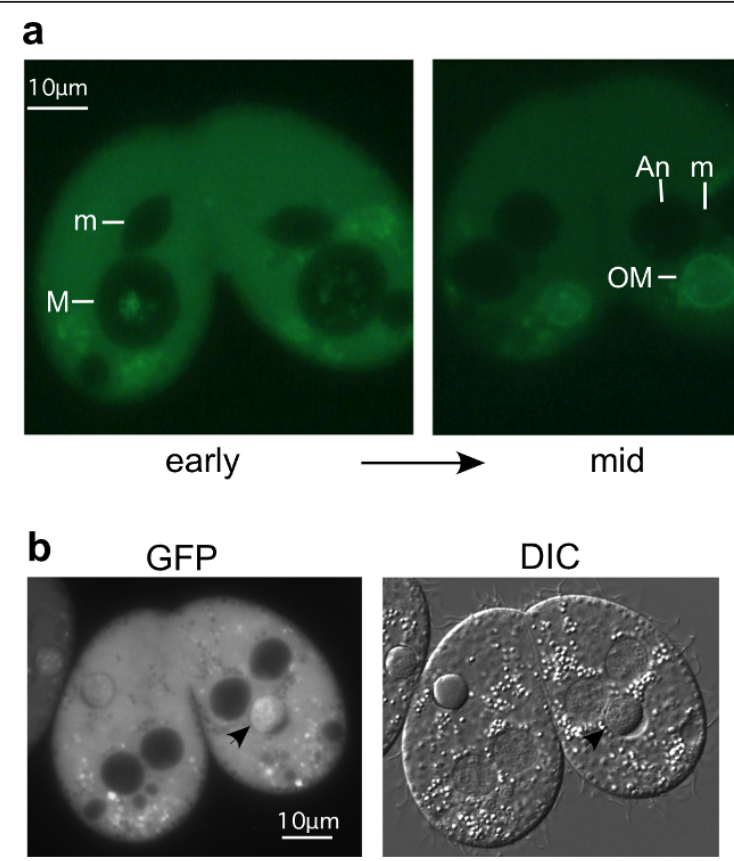

Figure 8 Thd 14 concentrates inside the macronucleus immediately prior to pycnosis. a Fluorescence microscopy images of live conjugating pairs showing localization of GFP-Thd14 at three points in conjugation designated "early" (before meiosis), "mid" (after meiosis, middevelopment of anlagen) and "late" (after pair separation, prior to macronuclear reabsorption). M, macronucleus; An, anlagen; m, micronucleus. Live cells were concentrated and mounted in $2 \%$ methylcellulose. $\mathbf{b}$ DIC and fluorescence microscopy images of a live conjugating pair showing GFP concentration over condensed chromatin regions in degrading macronuclei (arrows). Cells were prepared as in part a.

Unlike normal cells that lose their parental macronucleus by 16 hours into conjugation, NAM-treated cells retained a macronucleus that exhibited little to no DNA degradation (Figure 3). Programmed macronuclear degradation is thought to proceed through an apoptosislike, caspase-independent mechanism that is dependent on endonuclease $\mathrm{G}$ activity and apoptosis inducing factor (AIF) stored in mitochondria $[35,36]$. There is a well-described relationship in which chromatin condensation precedes DNA fragmentation in apoptosis [37]; one that also applies to programmed nuclear death in Tetrahymena $[35,38,39]$, but the mechanistic details underlying these chromatin changes in any system are poorly understood. In Tetrahymena, macronuclear DNA first condenses to less than half its original volume by 14 hours of conjugation, followed by global degradation of chromatin and resorption by the autophagosome $[13,35,39]$. This degree of condensation was inhibited in the majority of NAM-treated cells (Figure 1b). Given that DNA degradation also failed in these nuclei (Figure 3 ), sirtuin activity may be necessary for the chromatin condensation step of nuclear degradation, a prerequisite to global degradation. Interestingly, sirtuin inhibition did not affect the global deacetylation of histone $\mathrm{H} 4$ in parental macronuclear chromatin during pycnosis (Figure 2c), but it may be targeting other histones or nonhistone targets to mediate condensation. Our NAM treatment time course (Additional File 1) suggests sirtuin involvement in an earlier degradation step, possibly involving initial signaling. Normally, commitment of nuclei to new macronuclear development at 6-7 hours into conjugation triggers destruction of the old parental macronucleus, which involves its migration to the posterior region of the cell $[38,40]$. In our study, although NAMtreated nuclei committed to anlagen development, parental macronuclei failed to migrate in $\sim 30 \%$ of cells (Figures 1d and 3) consistent with possible disruption of the degradation "triggering" event.

Sirtuins are involved in signaling pathways preventing apoptosis and cellular senescence in other organisms [41], but no direct action on chromatin destined for degradation has been described. Although it is possible that the NAM treatment in our study inhibited nuclear degradation through blocking sirtuin-mediated signaling pathways, we present evidence that at least one Tetrahymena sirtuin, Thd14, could act directly on chromatin destined for degradation. Thd14 selectively accumulated in the parental macronucleus (not in anlagen) at the initial stage of chromatin condensation $(\sim 8 \mathrm{hrs})$, and in later stages mapped precisely over the region of condensed chromatin within the macronuclear envelope (Figures $8 \mathrm{a}$ and $8 \mathrm{~b}$ ). The mechanism for Thd14 
accumulation will be the focus of future studies. We speculate that Thd14 plays a role in macronuclear degradation by directly modifying histones. While our HDAC assay confirmed that Thd14 is capable of deacetylating histones in vitro (Additional File 3), it is also possible that this enzyme may target other substrates under biologically relevant conditions. Other than histone H2AX and $\mathrm{H} 2 \mathrm{~B}$ phosphorylation, little is known about other modifications to apoptotic chromatin, but histone deacetylation appears critical in at least some cases. Deacetylation of yeast histone H2B by the Hos3 HDAC (class II enzyme) is required for apoptosis [42], and apoptotic condensation in leukemia cells was linked with global histone deacetylation [43], but the deacetylases remain unknown. Given their involvement in heterochromatin formation at various genomic loci [6], sirtuins are reasonable candidates for apoptotic chromatin modifiers. Our combined results from NAM-inhibition and Thd14 localization provide the first evidence that a sirtuin(s) acts on chromatin destined for degradation.

One intriguing feature of Thd14 is its zinc finger domain, which is unusual to find on a sirtuin enzyme. Its strong homology to the PAZ domain on HDAC6 known to bind ubiquitin [23-25], suggests that Thd14 may interact with ubiquitin or ubiquitinated proteins, especially since it contains all of the essential binding residues [24] (Figure 4d). Since ubiquitin plays a major role in apoptosis and labels proteins for degradation, the PAZ and sirtuin domains of Thd14 may collaborate to make essential apoptotic modifications. Although a single protein with both a sirtuin and PAZ domain has not been identified in higher organisms, Thd14 may combine functions that higher-order organisms have evolved to handle with separate, more specialized proteins. To assess these possibilities, function of the putative PAZ domain and its potential role in nuclear degradation will be the subject of future studies.

Results in this study showed that Thd14 targeting was dependent on physiological state. In the nucleus of vegetatively growing cells, Thd 14 resided primarily in the multiple nucleoli positioned around the nuclear periphery, and in mitochondria. Sirtuins in other organisms are known to act at nucleolar loci where they stabilize rDNA repeats and factor into RNA polymerase I transcription and rDNA silencing [44-47]. Interestingly, nutrient starvation dramatically increased THD14 expression (Figure $5 \mathrm{c}$ ), and caused Thd14 protein to concentrate into a prominent focus, or aggregate, inside the nucleus (Figure 7a). Like nucleoli, this aggregate was often associated with the nuclear periphery and stained weakly with DAPI (Figure 7a, left panel). However, it was much larger than the typical nucleolar aggregates previously observed by electron microscopy $[48,49]$, and was ringed only around the periphery by the nucleolar protein Nop52 (Figure 7c).
Previous work showed that under certain gene overexpression conditions (PML and p53) the human Sir2 homolog, SIRT1, is recruited to discrete nuclear foci with promyelocytic leukemia (PML) protein, in "PML bodies" where it deacetylates proteins such as p53 [50]. Similarly, Thd14 may be sequestering with other nuclear proteins, possibly those involved in regulating cellular response to starvation stress.

Our study raises the intriguing possibility that the sirtuin Thd14 is specialized for the formation of irreversible heterochromatin functionally linked to parental macronucleus degradation, but not for reversible, facultative heterochromatin like meiotic chromatin in micronuclei. Despite the elevated expression in pre-meiotic stages, Thd14 did not localize to micronuclei at any point before or during meiosis, and formation of these two types of heterochromatin appears mechanistically different with respect to to histone modifications [51]. Especially intriguing is that localization results suggest additional roles for Thd14 in nucleoli and mitochondria. Whether they relate to nuclear degradation mechanisms will be a focus of future studies. In one possible model consistent with the macronuclear autophagy process, Thd14 is delivered specifically to the parental macronucleus from mitochondria that fuse with the nucleus prior to degradation, a mechanism previously shown to deliver other degradation factors such as endonucleases and AIF [35,52]. Such a model would explain the increased concentration of parental macronuclear Thd14 in the absence of increased expression (Figure $5 b)$. Regardless of its concentration mechanism, we expect future work to define a role for Thd14 in promoting or coordinating macronuclear autophagy.

\section{Conclusions}

The sirtuin inhibitor nicotinamide prevents meiotic chromosome condensation, and normal progression of both chromatin differentiation and programmed nuclear degradation during development in Tetrahymena, all of which depend on global chromatin condensation. One sirtuin, Thd14, resides in mitochondria, nucleoli, and in distinct nuclear sub-structures depending on physiological conditions and stages of the cell's life cycle. Notably, Thd14 accumulates in chromatin-rich regions of the degrading macronucleus during the chromatin condensation stage preceding global degradation. Together, the NAM-inhibition and Thd14 localization studies suggest the first evidence that sirtuins act on chromatin destined for degradation in apoptotic nuclei.

\section{Methods \\ Bioinformatics}

The amino acid sequences of the eleven Tetrahymena sirtuins (Thd8 through 18), yeast Sir2 and Hst1 through 
4, and human SIRT1 through 7 were aligned using Tree-based Consistency Objective Function For alignment Evaluation (TCOFFEE; http://www.ebi.ac.uk/t-coffee/). The molecular phylogeny was then evaluated using Multiple Sequence Alignment-CLUSTALW [Kyoto University Bioinformatics Center http://www. genome.jp/tools/clustalw/]. The CLUSTAL protein alignment was performed using a gap open penalty of 10 , a gap extension penalty of 0.05 , a hydrophobic gap, no weight transition, and a BLOSUM weight matrix. Distances were computed using the Poisson Correction Distance method in Molecular Evolutionary Genetic Analysis (MEGA) software version 4.0 (MEGA4) [53]. The unweighted-pair group method using an average linkages tree was constructed from the matrix of distances according to the model using MEGA4, and the robustness of the tree topology was tested with 1,000 bootstrap replicates. Sirtuin core domains were identified using ExPASY-PROSITE [Swiss Institute of Bioinformatics http://prosite.expasy.org/].

\section{Statistical analyses}

For qRT-PCR: results are indicated as the mean \pm standard deviation. Statistical significance in qRT-PCR and NAM treatment studies was determined by independent, two tailed $t$-tests in Microsoft Excel to compare differences between two groups. p-values of $<0.05$ were considered significant.

\section{Strains and cell culture conditions}

Tetrahymena thermophila strains CU727 (btu1-1::btu11M350K/btu1-1::btu1-1M350K; ory-r, tax-s, V), CU724 (btu1-1::btu1-1M350K/btu1-1::btu1-1M350K, chx1-1/ chx1-1; cy-r, mp-r, ory-r, tax-s, VII), CU427 (chx1-1/ chx1-1 CHX1; cy-s, VI), and CU428 (mpr1-1/mpr1-1 MPR1; mp-s, VII) provided by the National Tetrahymena Stock Center at Cornell University, were used as wild-type strains. For all experiments, Tetrahymena thermophila strains including the strain expressing GFPtagged Thd14 (GFP-Thd14) were grown in $2 \%$ PPYS medium $(0.02 \mathrm{~g} / \mathrm{mL}$ proteose peptone, $0.002 \mathrm{~g} / \mathrm{mL}$ yeast extract, and $0.03 \mathrm{mg} / \mathrm{mL}$ sequestrine) containing $2 \mathrm{X}$ PSF (penicillin, streptomycin, and fungizone; Gibco-BRL) with shaking $(150 \mathrm{rpm})$ at $30^{\circ} \mathrm{C}$, until mid-logarithmic phase $\left(1 \times 10^{5}\right.$ to $3 \times 10^{5}$ cells $\left./ \mathrm{ml}\right)$. The cells were starved in $10 \mathrm{mM}$ Tris- $\mathrm{HCl}(\mathrm{pH} 7.4)$ for 14 to $24 \mathrm{hrs}$ at a density of $3 \times 10^{5}$ cells $/ \mathrm{ml}$ at $30^{\circ} \mathrm{C}$ without shaking.

For nicotinamide (NAM) treatment experiments, cells of two different mating types (CU428 and CU427) were mixed together after starving each strain for 18-24 hrs in $10 \mathrm{mM}$ Tris ( $\mathrm{pH}$ 7.5). Cells were treated with $50 \mathrm{mM}$ NAM at the time of mixing ("0 hrs"), and with $25 \mathrm{mM}$ NAM at $0,2,4$, or $6 \mathrm{hrs}$ after mixing. For analysis of developmental morphology, at least 200 cells were counted for each time point and NAM concentration.

\section{HDAC activity assay}

Whole cell protein lysates were generated from $7 \times 10^{6}$ cells of both CU427 (wild type) and cells expressing HAThd14 by vortexing for $1 \mathrm{~min}$ with acid-washed glass beads (Sigma) in $350 \mu \mathrm{L}$ of lysis buffer ( $25 \mathrm{mM}$ Tris $\mathrm{pH}$ $8,15 \mathrm{mM} \mathrm{NaCl}, 10 \mathrm{mM} \mathrm{MgCl} 2,0.1 \mathrm{mM} \mathrm{CaCl}_{2}, 1 \mathrm{mM}$ phenylmethanesulfonyl fluoride, $0.05 \mathrm{mM}$ dithiothreitol). After treatment with DNase (277 U) for $20 \mathrm{~min}$, the lysates were clarified by centrifuging at 13,000 xg for 15 min and then were incubated with Ezview Red Anti-HA affinity gel (Sigma) for $1 \mathrm{hr}$ at $4^{\circ} \mathrm{C}$. The gel was washed 3 times with assay buffer ( $50 \mathrm{mM}$ Tris, $\mathrm{pH} 8,137 \mathrm{mM}$ $\mathrm{NaCl}, 2.7 \mathrm{mM} \mathrm{KCl}, 1 \mathrm{mM} \mathrm{MgCl}{ }_{2}$ ) and resuspended in the same buffer to yield a total volume of $60 \mu \mathrm{L}$.

Assays were performed in a 96 well plate with the HDAC Fluorometric Assay/Drug Discovery Kit (Enzo Life Sciences). Each $50 \mu \mathrm{L}$ assay contained $1 \mu \mathrm{M}$ Trichostatin A (type I and II HDAC inhibitor), $20 \mu \mathrm{L}$ of the gellysate slurry, $200 \mu \mathrm{M}$ nicotinamide adenine dinucleotide $\left(\mathrm{NAD}^{+}\right)$and $500 \mu \mathrm{M}$ Fluor de Lys ${ }^{\circledR}$ substrate in assay buffer. For the blank, 1 mM NAM was added before the substrate to inhibit any sirtuin activity. After $1 \mathrm{hr}$ at $37^{\circ} \mathrm{C}, 50$ $\mu \mathrm{L}$ of Fluor de Lys $^{\circledR}{ }^{\circledR}$ developer and $1 \mathrm{mM}$ NAM was added to quench the reaction. The fluorescence intensity of each assay was measured with a Perkin Elmer LS-55, Molecular Devices SpectraMax Gemini EM using an excitation of $355 \mathrm{~nm}$ and an emission of $460 \mathrm{~nm}$.

\section{Plasmid construction}

pIGF-GTW::THD14 for GFP-Thd14 expression. The THD14 gene was amplified from Tetrahymena genomic DNA by polymerase chain reaction (PCR) using forward primer 5'CACCATGAGTTCTGAAATTAGTAAAAC-3' and reverse primer 5'-TCAAAGGTTTTATTTCTTCTCTA-3'. The resulting PCR product was directionally cloned into plasmid pENTR/D-TOPO (Invitrogen) to make plasmid pENTR-THD14, and transformed into chemically competent TOP10 Escherichia coli cells (Invitrogen). After verification of the cloned THD14 sequence, the gene was exchanged with the Gateway cassette in pIGF-GTW [54] by combining 150 ng of pENTR-THD14 entry clone with 400 ng of pIGF-GTW and recombinase (LR Clonase II, Invitrogen) and incubating for $20 \mathrm{hrs}$ at $22^{\circ} \mathrm{C}$. Following proteinase $\mathrm{K}$ digestion, recombination reactions were electroporated into DH10B Escherichia coli made electrocompetent by a published method [55]. The resulting pIGF-GTW::THD14 plasmid contained the sequence construct to express a GFP fusion with the amino terminus of Thd14 under control of the MTT1 promoter. The construct was confirmed by sequencing. 
To make the plasmid for expression of HA-Thd14 fusion protein ( $p B M 2 H A:: T H D 14)$, the THD14 sequence with 630 bp upstream and 532 bp downstream of the gene was cloned into pCR-BluntII-TOPO vector (Invitrogen). SalI and AvrII restriction sites were introduced through site-directed mutagenesis (QuikChange, Stratagene) at the start codon and directly after the stop codon respectively (pTHD14SA). After digesting the plasmid with SalI and AvrII, the THD14 coding sequence was removed and then inserted into pBM2HA-YFG (vector containing the BTU1 flanking region surrounding the MTT1 promoter upstream of the multiple cloning sites and a sequence encoding a double HA peptide tag) for integration into the BTU1 locus of btu1-1 cells. The resulting plasmid was named " $p B M 2 H A-T H D 14$ ".

\section{Tetrahymena transformation}

The HA-THD14 fusion construct (from plasmid pBM2HA::THD14 digested with KpnI and SacI) was integrated into the taxol-sensitive beta-tubulin (btu1-1) locus of starved Tetrahymena cells (strain CU727) through biolistic bombardment as previously described [56]. Transformants were selected by growth in the presence of $20 \mu \mathrm{M}$ paclitaxel (MP Biomedicals) after 5 hrs of incubation at $30^{\circ} \mathrm{C}$ without drug. The construct for GFP tagging of THD14 (pIGF-GTW::THD14) was transformed into strain CU428 by electroporation according to a previously published method [57]. Transformants were selected by growth in the presence of $100 \mu \mathrm{g} / \mathrm{mL}$ paromomycin.

\section{Reverse-transcriptase PCR, quantitative PCR, and coding sequence determination}

Genomic DNA was isolated as described previously (61). Total RNA was isolated from vegetatively dividing, starved, and conjugating cells using the RNeasy Total RNA kit (Qiagen). The cDNA was made as previously described [56] using $2 \mu \mathrm{g}$ of total RNA for each reaction. The cDNA was used to perform qPCR using SsoFast EvaGreen supermix with low ROX (Bio-Rad) following the manufacturer's directions in a MJ MiniOpticon system (Bio-Rad). Genomic DNA dilutions were used for a standard curve and CYP1 or BTU1 primers were used for normalization of THD14 expression levels. Expression values were set relative to the CU428 starved value in the conjugation expression experiment and logarithmically growing cells in the starvation expression experiment. The following primer sets were used for qPCR; THD14 and CYP1 primers were designed to flank an intron in order to differentiate between products from cDNA or possible contaminating genomic DNA template for THD14:

THD14-QF (5'-CTGATTTGGTCGTCATGG-3')

THD14-QR (5'-ACAGTTCCTTCAGGGTATGTTC-3')

CYP1-QF (5'-AAGGATTAAGGTTAATGTGGTTCA-3')

CYP1-QR (5'-TTTCTGTACTGCAACATAGGGATA-3')
BTU1-QF (5'-GATAGAATCATGGAAACCTTCTC-3') BTU1-QR (5'-CAAGTGGTTAAGATCACCATAAG-3')

To determine the THD14 coding sequence, the entire coding sequence was amplified from cDNA using the primers for construction of pENTR-THD14 (above). The amplified sequence was subcloned onto pENTR plasmid (Invitrogen) and sequenced using M13-forward and M13-reverse primers. The coding sequence was submitted to Genbank, (accession number HQ156951).

\section{Immunoblot analysis}

Whole cell protein lysates were generated from $3 \times 10^{7}$ cells of both CU428 (wild type) and cells expressing HAThd14. Cells were lysed in $700 \mu \mathrm{L}$ of TLB $(350 \mathrm{mM}$ $\mathrm{NaCl}, 40$ mM HEPES pH 7.5, 1\% Triton X-100, 10\% glycerol, $1 \mathrm{mM}$ dithiothreitol), vortexed for 1 minute, and clarified by centrifuging at 13,000 xg for 15 minutes. Total protein concentration was determined using Bradford Reagent (Bio-Rad). Lysate (100 $\mu \mathrm{g}$ of total protein) was mixed with sodium dodecyl sulfate (SDS) gel loading buffer (50 mM Tris-HCl pH 6.8, $100 \mathrm{mM}$ dithiothreitol, $2 \%$ [wt/vol] SDS, $0.1 \%$ bromophenol blue, $10 \%$ glycerol) and boiled for $5 \mathrm{~min}$. Samples were loaded ( $20 \mu \mathrm{g}$ total protein per sample) and resolved by SDS-polyacrylamide gel electrophoresis (PAGE) on a 10\% polyacrylamide gel, transferred to nitrocellulose membrane, and incubated first with $\alpha$-HA antiserum (Covance) diluted 1:2,000 with $5 \%$ milk in Tris Buffered Saline (TBS). The membrane was washed $3 \times 5$ minutes in TBS and then incubated with horseradish peroxidase-conjugated goat $\alpha$-mouse antiserum (Pierce) diluted 1:10,000 with 5\% milk in TBS and developed using SuperSignal West Dura Chemiluminescence Kit (Pierce) and exposed to X-ray film.

\section{Microscopy}

For all live-cell imaging, $1 \mathrm{~mL}$ of culture was centrifuged at 2,000 $\mathrm{xg}$ and the pellet was incubated for $10 \mathrm{~min}$ with $0.1 \mu \mathrm{g}$ of 4,6 diamino-2-phenylindole dihydrochloride (DAPI; Sigma Chemicals). After dropping $5 \mu \mathrm{L}$ of $2 \%$ methylcellulose on a microscope slide, $1 \mu \mathrm{L}$ of the pellet was then added and covered with a \#1.5 micro cover slip (VWR). For mitochondrial imaging, cell cultures were incubated with $0.5 \mu \mathrm{g} / \mathrm{mL}$ Mito Tracker $^{\odot}$ Red CMXRos (Invitrogen) for at least 15 hrs and washed $3 \times$ with $10 \mathrm{mM}$ Tris Buffer ( $\mathrm{pH}$ 7.5) prior to sample preparation. Imaging involving mitochondria was performed on an Olympus $1 \times 81$ fluorescence microscope with a magnification of $100 \times$. All other fluorescence imaging was performed on a Nikon Eclipse E400 fluorescence microscope with a magnification of $40 \times$ or $100 \times$. For differential interference contrast (DIC) microscopy, cells were prepared in the same way as for fluorescence microscopy. 
Indirect immunofluorescence and DAPI staining of cells Growing and conjugating cells were fixed in 3\% paraformaldehyde and processed for immunofluorescence microscopy as previously described [58]. For detection of micronuclear linker histone (MLH), cells were incubated with $\alpha$-MLH antiserum (1:500; generous gift from David Allis). For detection of Nop52, cells were incubated with $\alpha$-Nop52 antiserum (1:20,000; generous gift from Ronald Pearlman). All primary antisera were detected with rhodamine-conjugated goat anti-rabbit immunoglobulin G (1:100; Jackson ImmunoResearch catalog no. 111-025003). Fixed cells were counterstained by incubation with $0.1 \mu \mathrm{g} / \mathrm{ml}$ DAPI in $0.1 \%$ bovine serum albumin-phosphate-buffered saline for $10 \mathrm{~min}$.

\section{TUNEL assay}

Conjugating cells (CU428 $\times$ CU427) were fixed $10 \mathrm{hrs}$ after mixing in $2 \%$ paraformaldehyde and stored in $70 \%$ ethanol at $-80^{\circ} \mathrm{C}$ for at least $24 \mathrm{hrs}$. The TUNEL (terminal deoxynucleotidyl transferase dUTP nick end labeling) assay was performed following the manufacturer's instructions for the APO-DIRECT kit (BD Pharmigen). In short, fixed cells were incubated overnight at $30^{\circ} \mathrm{C}$ in DNA labeling solution (containing FITC dUTP and TdT enzyme) and then exposed for $30 \mathrm{~min}$ at room temperature to Propidium Iodide/RNase staining buffer prior to imaging.

\section{Abbrevations}

GFP: green fluorescent protein; HDAC: histone deacetylase; NAM: nicotinamide; $\mathrm{NAD}^{+}$: nicotinamide adenine dinucleotide; MLH: micronuclear linker histone; MTT1: metallothioneine; OM: old macronucleus; PCR: polymerase chain reaction; TBS: Tris buffered saline; Thd14: Tetrahymena histone deacetylase 14; TUNEL: terminal deoxynucleotidyl transferase dUTP nick end labeling

\section{Additional material}

Additional file 1: "Time dependence of nicotinamide treatment effects". This data illustrates a decrease in the old macronucleus retention phenotype with NAM treatment later in conjugation.

Additional file 2: "Thd14 sirtuin core domain resembles that of other class I sirtuins". This is an alignment of Thd14 with the sirtuin domains of yeast and human enzymes. It shows strong conservation of active site residues and metal binding residues.

Additional file 3: "Thd14 has histone deacetylase activity". This is data from an experiment testing for histone deacetylase activity. It shows that Thd14 deacetylates histone amino-terminal peptides.

\section{Acknowledgements and Funding}

We are grateful for the contributions made by several colleagues: Douglas Chalker for performing DIC imaging and for many helpful discussions, C. David Allis for providing anti-MLH antiserum, Ronald Pearlman for providing anti-Nop52 antiserum, Allen Chen for imaging starved cells, Linda Wang for initial work on nicotinamide treatment experiments, and Lindsey Moneta for assistance with constructing GFP-Thd14. This work was supported by National Science Foundation grant \#0545560 made to E.A.W.

\section{Author details}

${ }^{1}$ Keck Science Department of Claremont McKenna, Pitzer, and Scripps Colleges, W.M. Keck Science Center, 925 N. Mills Ave., Claremont, CA 91711, USA. ${ }^{2}$ Chemistry Department, Hobart and Williams Smith College, 300 Pultney St., Geneva, NY 14456, USA. ${ }^{3}$ Biomedical Sciences Department, Missouri State University, 901 S. National Ave., Springfield, MO 65897, USA.

\section{Authors' contributions}

KMS performed the TUNEL assays, NAM experiments, HDAC assay, GFPThd14/mitotracker microscopy, and helped draft and revise the manuscript. SF produced the genetic constructs and transformed cell lines, initial gene expression profiles, GFP-Thd14 nuclear localization and Nop52 immunofluorescence results, and performed some of the bioinformatics analyses. KAC performed the GRT-PCR, some bioinformatics analyses, all statistics, and helped revise the manuscript. JJS assisted with transformations, helped draft and revise the manuscript, and assisted with supervision of experiments. EAW conceived the idea, drafted the manuscript, supervised the experiments, and performed some of the imaging. All authors read and approved the final manuscript.

\section{Competing interests}

The authors declare that they have no competing interests.

Received: 1 April 2011 Accepted: 21 September 2011

Published: 21 September 2011

\section{References}

1. Frye RA: Phylogenetic classification of prokaryotic and eukaryotic Sir2like proteins. Biochemical and biophysical research communications 2000, 273(2):793-798.

2. Saunders LR, Verdin E: Sirtuins: critical regulators at the crossroads between cancer and aging. Oncogene 2007, 26(37):5489-5504.

3. Michan S, Sinclair D: Sirtuins in mammals: insights into their biological function. The Biochemical journal 2007, 404(1):1-13.

4. North BJ, Marshall BL, Borra MT, Denu JM, Verdin E: The human Sir2 ortholog, SIRT2, is an NAD+-dependent tubulin deacetylase. Molecular cell 2003, 11(2):437-444.

5. Dryden SC, Nahhas FA, Nowak JE, Goustin AS, Tainsky MA: Role for human SIRT2 NAD-dependent deacetylase activity in control of mitotic exit in the cell cycle. Molecular and cellular biology 2003, 23(9):3173-3185.

6. Vaquero $A$ : The conserved role of sirtuins in chromatin regulation. The International journal of developmental biology 2009, 53(2-3):303-322.

7. Finkel T, Deng CX, Mostoslavsky R: Recent progress in the biology and physiology of sirtuins. Nature 2009, 460(7255):587-591.

8. Donmez G, Guarente L: Aging and disease: connections to sirtuins. Aging cell 9(2):285-290

9. Smith JJ, Torigoe SE, Maxson J, Fish LC, Wiley EA: A class II histone deacetylase acts on newly synthesized histones in Tetrahymena. Eukaryotic cell 2008, 7(3):471-482.

10. Wiley EA, Myers T, Parker K, Braun T, Yao MC: Class I histone deacetylase Thd $1 \mathrm{p}$ affects nuclear integrity in Tetrahymena thermophila. Eukaryotic cell 2005, 4(5):981-990.

11. Jeter JR Jr, Pavlat WA, Cameron IL: Changes in the nuclear acidic proteins and chromatin structure in starved and refed tetrahymena. Experimental cell research 1975, 93(1):79-88.

12. Cole ES, Frankel J: Conjugal blocks in Tetrahymena pattern mutants and their cytoplasmic rescue. II. janus A. Developmental biology 1991, 148(2):420-428.

13. Davis MC, Ward JG, Herrick G, Allis CD: Programmed nuclear death: apoptotic-like degradation of specific nuclei in conjugating Tetrahymena. Developmental biology 1992, 154(2):419-432.

14. Lu C, Zhu F, Cho YY, Tang F, Zykova T, Ma WY, Bode AM, Dong Z: Cell apoptosis: requirement of $\mathrm{H} 2 \mathrm{AX}$ in DNA ladder formation, but not for the activation of caspase-3. Molecular cell 2006, 23(1):121-132.

15. Artus C, Boujrad H, Bouharrour A, Brunelle MN, Hoos S, Yuste VJ, Lenormand P, Rousselle JC, Namane A, England P, et al: AIF promotes chromatinolysis and caspase-independent programmed necrosis by interacting with histone H2AX. The EMBO journal 2010, 29(9):1585-1599. 
16. Grubisha O, Smith BC, Denu JM: Small molecule regulation of Sir2 protein deacetylases. The FEBS journal 2005, 272(18):4607-4616.

17. Bitterman KJ, Anderson RM, Cohen HY, Latorre-Esteves M, Sinclair DA: Inhibition of silencing and accelerated aging by nicotinamide, a putative negative regulator of yeast sir2 and human SIRT1. The Journal of biological chemistry 2002, 277(47):45099-45107.

18. Denu JM: Linking chromatin function with metabolic networks: Sir2 family of $\mathrm{NAD}(+)$-dependent deacetylases. Trends in biochemical sciences 2003, 28(1):41-48.

19. Duharcourt S, Yao MC: Role of histone deacetylation in developmentally programmed DNA rearrangements in Tetrahymena thermophila. Eukaryotic cell 2002, 1(2):293-303.

20. Scherthan H: A bouquet makes ends meet. Nature reviews 2001, 2(8):621-627.

21. Loidl J, Scherthan H: Organization and pairing of meiotic chromosomes in the ciliate Tetrahymena thermophila. Journal of cell science 2004, 117(Pt 24):5791-5801.

22. Chicoine $L G$, Wenkert $D$, Richman R, Wiggins JC, Allis CD: Modulation of linker histones during development in Tetrahymena: selective elimination of linker histone during the differentiation of new macronuclei. Developmental biology 1985, 109(1):1-8

23. Hook SS, Orian A, Cowley SM, Eisenman RN: Histone deacetylase 6 binds polyubiquitin through its zinc finger (PAZ domain) and copurifies with deubiquitinating enzymes. Proceedings of the National Academy of Sciences of the United States of America 2002, 99(21):13425-13430.

24. Boyault C, Gilquin B, Zhang Y, Rybin V, Garman E, Meyer-Klaucke W, Matthias P, Muller CW, Khochbin S: HDAC6-p97/NCP controlled polyubiquitin chain turnover. The EMBO journal 2006, 25(14):3357-3366

25. Lee JY, Koga H, Kawaguchi Y, Tang W, Wong E, Gao YS, Pandey UB, Kaushik S, Tresse E, Lu J, et al: HDAC6 controls autophagosome maturation essential for ubiquitin-selective quality-control autophagy. The EMBO journal 2010, 29(5):969-980.

26. Miao W, Xiong J, Bowen J, Wang W, Liu Y, Braguinets O, Grigull J, Pearlman RE, Orias E, Gorovsky MA: Microarray analyses of gene expression during the Tetrahymena thermophila life cycle. PloS one 2009, 4(2):e4429.

27. Yao MC, Yao CH: Transformation of Tetrahymena to cycloheximide resistance with a ribosomal protein gene through sequence replacement. Proceedings of the National Academy of Sciences of the United States of America 1991, 88(21):9493-9497.

28. Boldrin F, Santovito G, Gaertig J, Wloga D, Cassidy-Hanley D, Clark TG, Piccinni E: Metallothionein gene from Tetrahymena thermophila with a copper-inducible-repressible promoter. Eukaryotic cell 2006, 5(2):422-425.

29. McGrath KE, Smothers JF, Dadd CA, Madireddi MT, Gorovsky MA, Allis CD: An abundant nucleolar phosphoprotein is associated with ribosomal DNA in Tetrahymena macronuclei. Molecular biology of the cell 1997, 8(1):97-108

30. Satir B, Dirksen ER: Nucleolar aging in Tetrahymena during the cultural growth cycle. The Journal of cell biology 1971, 48(1):143-154.

31. Sugai T, Hiwatashi K: Cytologic and autoradiographic studies of the micronucleus at meiotic prophase in Tetrahymena pyriformis. The Journal of protozoology 1974, 21(4):542-548.

32. Mochizuki K, Novatchkova M, Loidl J: DNA double-strand breaks, but not crossovers, are required for the reorganization of meiotic nuclei in Tetrahymena. Journal of cell science 2008, 121(Pt 13):2148-2158.

33. Vaquero $A$, Scher MB, Lee DH, Sutton $A$, Cheng HL, Alt FW, Serrano L, Sternglanz R, Reinberg D: SirT2 is a histone deacetylase with preference for histone H4 Lys 16 during mitosis. Genes \& development 2006, 20(10):1256-1261.

34. Yakisich JS, Kapler GM: The effect of phosphoinositide 3-kinase inhibitors on programmed nuclear degradation in Tetrahymena and fate of surviving nuclei. Cell death and differentiation 2004, 11(10):1146-1149.

35. Akematsu T, Endoh H: Role of apoptosis-inducing factor (AIF) in programmed nuclear death during conjugation in Tetrahymena thermophila. BMC cell biology 2010, 11:13.

36. Kobayashi T, Endoh H: A possible role of mitochondria in the apoptoticlike programmed nuclear death of Tetrahymena thermophila. The FEBS journal 2005, 272(20):5378-5387.

37. Tone $S$, Sugimoto K, Tanda K, Suda T, Uehira K, Kanouchi H, Samejima K, Minatogawa Y, Earnshaw WC: Three distinct stages of apoptotic nuclear condensation revealed by time-lapse imaging, biochemical and electron microscopy analysis of cell-free apoptosis. Experimental cell research 2007, 313(16):3635-3644.

38. Mpoke S, Wolfe J: DNA digestion and chromatin condensation during nuclear death in Tetrahymena. Experimental cell research 1996, 225(2):357-365.

39. Kobayashi T, Endoh H: Caspase-like activity in programmed nuclear death during conjugation of Tetrahymena thermophila. Cell death and differentiation 2003, 10(6):634-640.

40. Lu E, Wolfe J: Lysosomal enzymes in the macronucleus of Tetrahymena during its apoptosis-like degradation. Cell death and differentiation 2001, 8(3):289-297.

41. Liu T, Liu PY, Marshall GM: The critical role of the class III histone deacetylase SIRT1 in cancer. Cancer research 2009, 69(5):1702-1705.

42. Ahn SH, Diaz RL, Grunstein M, Allis CD: Histone H2B deacetylation at lysine 11 is required for yeast apoptosis induced by phosphorylation of H2B at serine 10. Molecular cell 2006, 24(2):211-220.

43. Boix-Chornet M, Fraga MF, Villar-Garea A, Caballero R, Espada J, Nunez A, Casado J, Largo C, Casal Jl, Cigudosa JC, et al: Release of hypoacetylated and trimethylated histone $\mathrm{H} 4$ is an epigenetic marker of early apoptosis. The Journal of biological chemistry 2006, 281(19):13540-13547.

44. Ford E, Voit R, Liszt G, Magin C, Grummt I, Guarente L: Mammalian Sir2 homolog SIRT7 is an activator of RNA polymerase I transcription. Genes \& development 2006, 20(9):1075-1080.

45. Haigis MC, Guarente LP: Mammalian sirtuins-emerging roles in physiology, aging, and calorie restriction. Genes \& development 2006, 20(21):2913-2921.

46. Straight AF, Shou W, Dowd GJ, Turck CW, Deshaies RJ, Johnson AD, Moazed D: Net1, a Sir2-associated nucleolar protein required for rDNA silencing and nucleolar integrity. Cell 1999, 97(2):245-256.

47. Moazed D: Common themes in mechanisms of gene silencing. Molecular cell 2001, 8(3):489-498.

48. Cameron IL, Guile EE: Nucleolar and Biochemical Changes During Unbalanced Growth of Tetrahymena Pyriformis. The Journal of cell biology 1965, 26(3):845-855.

49. Elliott AM, Kennedy JR Jr, Bak IJ: Macronuclear events in synchronously dividing Tetrahymena pyriformis. The Journal of cell biology 1962, 12:515-531.

50. Langley E, Pearson M, Faretta M, Bauer UM, Frye RA, Minucci S, Pelicci PG, Kouzarides T: Human SIR2 deacetylates p53 and antagonizes PML/p53induced cellular senescence. The EMBO journal 2002, 21(10):2383-2396.

51. Ajiro K: Histone $\mathrm{H} 2 \mathrm{~B}$ phosphorylation in mammalian apoptotic cells. An association with DNA fragmentation. The Journal of biological chemistry 2000, 275(1):439-443.

52. Akematsu T, Pearlman RE, Endoh H: Gigantic macroautophagy in programmed nuclear death of Tetrahymena thermophila. Autophagy 2010, 6(7):901-911

53. Tamura K, Dudley J, Nei M, Kumar S: MEGA4: Molecular Evolutionary Genetics Analysis (MEGA) software version 4.0. Molecular biology and evolution 2007, 24(8):1596-1599.

54. Malone CD, Falkowska KA, Li AY, Galanti SE, Kanuru RC, LaMont EG, Mazzarella KC, Micev AJ, Osman MM, Piotrowski NK, et al: Nucleus-specific importin alpha proteins and nucleoporins regulate protein import and nuclear division in the binucleate Tetrahymena thermophila. Eukaryotic cell 2008, 7(9):1487-1499.

55. Sambrook J, Russell D: Transformation of E. coli by Electroporation. In Molecular Cloning: A Laboratory Manual. Volume 1.. 3 edition. Cold Spring Harbor, NY: Cold Spring Harbor Laboratory Press; 2001:120-121.

56. Marsh TC, Cole ES, Romero DP: The transition from conjugal development to the first vegetative cell division is dependent on RAD51 expression in the ciliate Tetrahymena thermophila. Genetics 2001, 157(4):1591-1598.

57. Asai DJ, Forney JD: Tetrahymena thermophila. In Methods in cell biology. Volume 62. Edited by: L W, P M. San Diego, CA Academic Press; 2000:489-490.

58. Cole ES, Stuart KR, Marsh TC, Aufderheide K, Ringlien W: Confocal fluorescence microscopy for Tetrahymena thermophila. Methods in cell biology 2002, 70:337-359.

doi:10.1186/1471-2121-12-40

Cite this article as: Slade et al:: Sirtuin-mediated nuclear differentiation and programmed degradation in Tetrahymena. BMC Cell Biology 2011 12:40. 